\title{
Predicting hospice appropriateness for nursing home residents with dementia in Korea: $A$ systematic review approach
}

\author{
Juh Hyun Shin * \\ Division of Nursing Science, College of Health Sciences, Ewha Womans University, Korea
}

Received: July 24, 2014

DOI: $10.5430 / \mathrm{cns} . \mathrm{v} 3 \mathrm{n} 1 \mathrm{p} 14$
Accepted: September 1, $2014 \quad$ Online Published: November 5, 2014

URL: http://dx.doi.org/10.5430/cns.v3n1p14

\begin{abstract}
Background: Korea is one of the fastest aging societies; the number of elderly who die due to Alzheimer dementia has increased dramatically. The benefits of hospice care were recognized in effective pain management, advance-care planning, decreased hospitalization, decreased aggressive treatment, and increased job satisfaction. To estimate life expectancy of residents with dementia precisely is difficult, exacerbating the ability to provide palliative care for residents with dementia. However, the underuse of hospice care in nursing homes (NHs) is a major problem in Korea. Few researchers have identified the needs of people dying with dementia in NHs in Korea. The purpose of this paper was to review and discuss hospice-eligibility assessment tools.

Methods: Four tools were available for discussion at the point of investigation: the Functional Assessment Staging Scale, Alzheimer's Hospice Placement Evaluation Scale, Advanced Dementia Prognostic Tool from the U.S. Hospice Medicare guidelines, and Global Deterioration Scale.

Results: Major problems are underuse of hospice care and lack of guidelines to assess hospice in patients with dementia in Korea.

Conclusion: Successfully categorizing elders with dementia as hospice enrollees in Korea will improve the quality of end-of-life care for increasing numbers of elders with dementia and their family members.
\end{abstract}

Key Words: Elder dementia, Hospice-eligibility assessment, Functional assessment, Prognostic tool

\section{Introduction}

Korea is one of the fastest aging societies, having about $5,184,000$ elderly (about $11 \%$ of the total population). ${ }^{[1]}$ The prevalence of elders with dementia is about $9.18 \%$ of the total elderly population. ${ }^{[1]}$ By 2050, about 271,000 elderly will be diagnosed with dementia. Dementia incidence rates are expected to increase by $308 \%$ with a $503.7 \%$ healthcare-cost increase in Korea. ${ }^{[1,2]}$ The number of elderly who died from Alzheimer dementia increased from 1,393 (165 death rates per 10,000) in $2009^{[3]}$ to $2,700(260.3$ death rates per 10,000) in 2012. Also, deaths in residential settings like nursing homes (NHs) doubled from 4,884 deaths in $2009^{[3]}$ to 10,233 deaths in 2012. As of the end of 2012, about 118,631 residents lived in 2,610 NHs, and about $20.8 \%$ of residents in NHs suffer from demen-

\footnotetext{
*Correspondence: Juh Hyun Shin; Email: juhshin@ewha.ac.kr; Address: Division of Nursing Science, College of Health Sciences, Ewha Womans University, Seoul 120-750, Korea.
} 
tia in Korea. ${ }^{[1]}$ Only beneficiaries screened by a care-needassessment screening test by Korean National Health Insurance Corporation experts are eligible to be admitted to NHs; these are elders over 65 or disabled who need assistance with activities of daily living. ${ }^{[4]}$ As the number of elders with dementia increased, the Korean government lowered the scores of care-need-assessment screening tests to admit more dementia residents after July 2013. ${ }^{[3]}$ Furthermore, a national dementia-management system is operating for elderly residents with dementia in NHs who do not have national health insurance, to increase health promotion and decrease healthcare costs for dementia residents. ${ }^{[3]}$

Hospice care is intended to support comfort, quality of life, and death with dignity for patients and their families. ${ }^{[5]}$ Foreign missionaries and religious organizations initiated hospice care and are quite involved in hospice care in Korea. ${ }^{[6]}$ Reviews of literature supported the benefits of hospice care for those with dementia and their families, and professionals and caregivers recognized the benefits accruing in effective pain management, advance-care planning, decreased hospitalization, decreased aggressive treatments, and increased job satisfaction by long-term-care-facilities' staff. ${ }^{[7-10]}$ In a recent study, NH residents with dementia under the hospice program were more likely to have regular pain killers and appropriate symptom management in the last week before death. ${ }^{[7]}$ Also, after patients died, proxies reported high levels of satisfaction about hospice care for patients with dementia. ${ }^{[11]}$ However, most research about palliative care has focused on end-stage cancer patients, ${ }^{[6]}$ and the awareness of hospice programs among dementia patients and proxies was much lower (13\%) than that of cancer patients $(87 \%) .{ }^{[12]} \mathrm{NH}$ residents with dementia are less likely to get hospice care than those with cancer in NHs. ${ }^{[13]}$ Several U.S. national studies reported that $80 \%$ of hospices reported difficulty predicting survival, presenting a major problem to enrollees with dementia. ${ }^{[14]}$

Estimating residents with dementia's life expectancy precisely is difficult, exacerbating the difficulty of providing palliative care for residents with dementia. ${ }^{[15]}$ Adequate optimal palliative care is not provided through the final phase of illness of residents with dementia because dementia is usually not considered a terminal condition. ${ }^{[11]}$ Life expectancy for people with dementia is estimated to be approximately 4.5 years; consequently, hospice care for people with dementia may be inadequate, unsuitable, or undesirable. ${ }^{[17]}$ An accurate estimation of prognosis is important to increase the use of hospice for residents with dementia. NHs play a critical role in providing palliative care for residents with dementia. However, the underuse of hospice care in NHs tends to be a major problem in Korea. ${ }^{[18]}$ Reasons include the deficiency of awareness of hospice care, space, and financial difficulties. ${ }^{[18]}$ Moreover, residents in NHs have more anxiety and fear about death than elders at home because there are no family members in NHs. ${ }^{[19]}$ Conse- quently, hospice care is not performed, despite the need of most dying residents for holistic care at the end of life in NHs in Korea. Although, some published assessment tools exist to predict 6-month survival for dementia residents in NHs in the United States, little research identifies the needs of dying people with dementia in NHs in Korea. The purpose of this paper is to review and discuss hospice-eligibility assessment tools.

\section{Methods}

\subsection{Search strategy}

The study design was a systematic review. An online search of the electronic bibliographic databases included MEDLINE, Cumulative Index to Nursing and Allied Health Literature (CINAHL), OVID, and PUBMED (EBSCOhost and OVID) using the keywords elderly, dementia, hospice, screening, evaluation, and eligibility. The search included Riss4u for articles published in Korea. Keywords were searched individually and in combination with the others. First, journal articles which had been published after 2010 were selected; then, articles as old as 1980 were accepted and reviewed if they described the only published study available that met the following inclusion criteria. Each database was monitored monthly, with the final search conducted on August 20, 2014 to ensure the most current research articles. The decision flow chart is shown in Figure 1.



Figure 1: Systematic review of hospice eligibility for elderly with dementia flow chart

\subsection{Inclusion/exclusion criteria}

Inclusion criteria included original research, psychometric testing, and review articles published in English and Korean. For hospice-eligibility assessment-tool information, a search was conducted through Google Scholar. The population included only elders with dementia, and excluded cancer patients and general elderly. A total of 12,397 titles, using hospice as a keyword, were retrieved from the search 
of electronic bibliographic databases; also, 5,786 titles were retrieved using eligibility as the keyword. Combining these two keywords yielded a total of 178 articles. Of those, 63 were outdated or irrelevant and were excluded. Included were a total of 28 full-text articles that assessed eligibility of hospice for elders with dementia. Google Scholar yielded four additional articles. Theses, dissertations, and gray literature were excluded and only available full-text papers and instruments written in English or Korean were included. Ultimately, 30 articles were selected and included in this review, after reviewing abstracts and full texts where available. Included papers had to report primary research and have discussed or tested psychometric characteristics of a hospice-eligibility tool. Papers that screened solely for a hospice-eligibility assessment tool for $\mathrm{NH}$ residents with dementia were included.

\section{Results}

Analysis of these 30 articles revealed that only four tools were available for discussion at the point of investigation: the Global Deterioration Scale (GDS), the Functional Assessment Staging Scale (FAST), Alzheimer's Hospice Placement Evaluation Scale (AHOPE), and the U.S. Hospice Medicare guidelines' Advanced Dementia Prognostic Tool (ADEPT). Hospice services are available to those who have a 6-month survival prognosis and are eligible for Medicare and Medicaid in the United States. These programs do not exist in Korea. ${ }^{[20]}$

\subsection{Functional assessment staging scale}

The FAST is used widely worldwide to measure the level of cognitive impairment for people with dementia. ${ }^{[21]}$ According to the current National Hospice Palliative Care Organization Guidelines, ${ }^{[22]}$ two categories exist in the current hospice guidelines for patients with Alzheimer's disease (AD): functional assessment staging and the presence of comorbid medical complications. ${ }^{[22]}$ Researchers used National Hospice Organization (NHO) guidelines to create the FAST. ${ }^{[23]}$ The FAST scale is used to assess functional change in persons with dementia, composed of seven major stages (1-7f) with 16 substages. ${ }^{[23]}$ Patients who have Stage 7 in the FAST (those who are entirely dependent in all activities of daily living, are nonambulatory, and are unable to speak) are eligible for hospice care in the United States. ${ }^{[22]}$ Stage 7 corresponds to those with advanced AD and includes symptoms such as progressive loss of speech and locomotion and increasing levels of disability; Stages 16 should have been met previously. ${ }^{[22]}$ The major benefit of the FAST is that it can be applied to patients with dementia and end-stage AD. ${ }^{[24]}$

However, the FAST has been criticized because it does not precisely predict 6-month mortality. ${ }^{[16,23]}$ Many studies reported that the FAST did not originate from empirical data, and cannot apply to the majority of elders with dementia whose disease did not advance linearly. ${ }^{[15]}$ Furthermore, the FAST does not consider comorbid conditions such as pneumonia, upper urinary-tract infections, and swallowing difficulties. ${ }^{[7,25,26]}$ Consequently, the best prediction of a 6month prognosis can be accomplished by the combination of functional or cognitive assessments of disease severity and comorbid symptoms including aspiration pneumonia, upper urinary-tract infection, Stage III or IV pressure ulcers, repeated fever despite antibiotics, and nutritional problems. ${ }^{[27]}$

Mitchell and colleagues ${ }^{[26]}$ compared elderly residents who died with $\mathrm{AD}(\mathrm{N}=1,609)$ and terminal cancer $(\mathrm{N}=883)$ within 1 year of admission to New York State NHs using the Minimum Data Set (MDS). Major findings were (1) only $1.1 \%$ with $\mathrm{AD}$ were recognized to have a life expectancy less than 6 months but $71 \%$ died within that period; (2) $55.1 \%$ of residents with dementia had no Do Not Resuscitate orders and $1.4 \%$ had Do Not Hospitalize orders; (3) nonpalliative interventions generally included tube feeding $(25 \%)$, laboratory tests $(49.2 \%)$, restraints $(11.2 \%)$, and intravenous therapy $(10.1 \%)$; (4) residents with dementia were less likely than those with cancer to have directives restricting aggressive care but were more likely than those with cancer to experience burdensome interventions such as Do Not Resuscitate orders, tube feedings, laboratory tests, and restraints; and (5) residents with dementia had higher rates of pressure ulcers (14.7\%), constipation (13.7), pain $(11.5 \%)$, and shortness of breath $(8.2 \%){ }^{[28]}$ Mitchell, et al. ${ }^{[26]}$ also studied factors related to 6-month survival rates for newly admitted NH residents with dementia and risk scores to predict survival for this population. This study was a retrospective cohort study from the MDS in New York between 1994 and 1998 (derivation cohort, $N=6,799$ ) and Michigan between 1998 and 2000 (validation cohort, $\mathrm{N}=$ 4,631) of Medicare and Medicaid NH residents. MDS factors with 6-month mortality were verified in the derivation group whereas the consequential risk score was assessed in the validation cohort. Regarding the main outcome measures of this study, the cut point of 7c of the FAST was compared with the risk-score performance. This study reported that 6-month mortality of a risk score of 12 identified variables from the MDS better than the existing guidelines. ${ }^{[26]}$

Also, the clinical usefulness of the FAST has been tested in Korea. The Pearson correlations between the FAST and the Mini-Mental State Examination (MMSE) for the elderly with normal aging and Alzheimer's dementia $(n=50)$ have been reported to be good $(87 ; 24)$. The validity of the FAST was tested for patients with Alzheimer dementia in Korea, with the Korean MMSE $(r=-.71, p<.001)$, severe impairment battery $(r=-.54, p<.001)$, and the Baylor Profound Mental Status Examination $(r=-.46, p<.001$; Na, et al., 2010). In this study, the FAST was supported as a reliable and valid tool to assess the functional deterioration and 
the progression of Korean elderly throughout Alzheimer dementia. ${ }^{[29]}$

\subsection{Alzheimer's hospice placement evaluation scale}

One tool to assess the 6-month prognosis of individuals with end-stage Alzheimer-type dementia is the AHOPE, developed and validated by Marsh, et al. ${ }^{[20]}$ The AHOPE is a practical assessment tool developed to improve the prediction of hospice eligibility for AD elderly. The AHOPE evaluates symptom severity on nine indicators; each item consists of item-specific response choices based on a literature review, review of symptom-severity measures, and professionals' opinion. Scores range from 9 to 36 with higher scores indicating greater severity of symptoms. Six items are scored by assessing patients whereas three are scored from patient records. The nine indicators are level of consciousness, eye-contact, speech, muscle flexibility, ambulation, swallowing, food intake, fluid intake, and weight Among these items, swallowing, fluid intake, food intake, and weight change came from NHO guidelines. ${ }^{[20]}$

Marsh, et al. ${ }^{[20]}$ tested the reliability and predictive validity of the AHOPE by comparing data between data collectors and clinical experts and added demographic and clinical indicators to identify the appropriateness of hospice care using two logistic-regression models. The sample was 112 longterm-care residents with end-stage $\mathrm{AD}$ at enrollment and their next 6-month enrollment. Their work supported the initial reliability and predictive validity of the AHOPE, but did not support demographic and clinical indicators. In this study, Marsh and colleagues ${ }^{[20]}$ discussed additional items including loss of ability to communicate adequately, smile, and acknowledge loved ones. These items are consistent with NHO guidelines and criteria by Luchins, et al. ${ }^{[15]}$ Registered nurses can use AHOPE to enhance clinical observations and implement proper strategies for $\mathrm{AD}$ patients and their families, although additional research is necessary. ${ }^{[20]}$

\subsection{U.S. hospice medicare guidelines}

In 1996, the scope of eligibility in the hospice Medicare guideline was expanded beyond patients with cancer to those with dementia. ${ }^{[22]}$ Schonwetter and colleagues ${ }^{[30]}$ investigated the validity of Medicare hospice-eligibility guidelines and predictor issues for dementia patients. They reviewed retrospective medical records, including initial assessments and follow-up meetings until death $(\mathrm{N}=245)$ in a large hospice-care facility. They found no significant relationship between Medicare guidelines and 6-month survival; researchers suggested new empirically driven predictors and highlighted the importance of assessing comorbid diseases when predicting 6-month survival for dementia patients. ${ }^{[30]}$ Researchers continue to criticize the accuracy of the hospice Medicare guideline. ${ }^{[26,30]}$ as the inaccuracies of prognosis for hospice eligibility may result in the underuse or overuse of hospice care for elders with dementia, raising quality-of-life and cost issues. ${ }^{[31]}$

\subsection{Advanced dementia prognostic tool}

The National Institutes of Health in the United States developed the ADEPT tool to validate hospice-enrollment eligibility for AD people using the MDS, which is the mandated assessment tool for $\mathrm{NH}$ residents. ${ }^{[26]}$ Indicators include $\mathrm{NH}$ stay, age, sex, shortness of breath, pressure ulcers, activities of daily living, bedfastness, oral intake, bowel incontinence, body-mass index, weight loss, and congestive heart failure. ${ }^{[32]}$ Scores range from 1.0 to 32.5 with higher scores indicating greater risk of death. ${ }^{[32]}$ The ADEPT scale categorizes moderate hospice eligibility, interrater reliability, and sensitivity, each reported to have good validity; discrimination was modest and specificity was low. ${ }^{[9]}$

\subsection{Global deterioration scale}

Researchers developed the GDS to assess the seven stages of cognitive function, with a 7-item Likert scale indicating 1 (no cognitive impairment) to 7 (late severe cognitive impairment). ${ }^{[33]}$ Psychometric testing for Korean elders was supported. ${ }^{[34]}$ However, very highly trained experts should administer the $\operatorname{GDS}^{[35]}$ and the application to NHs may be limited; concrete education for staff is required. This scale partitions AD into 7 phases (Phase 1: no cognitive decline, Phase 2: very mild cognitive decline, Phase 3: Mild cognitive decline, Phase 4: Moderate cognitive decline, Phase 5: Moderately severe cognitive decline, Phase 6: Severe cognitive decline, Phase 7: Very severe cognitive decline). ${ }^{[33]}$ The major advantage of the GDS is to evaluate cognitive impairment and functional deterioration in NH residents. ${ }^{[36]}$ For elders with AD, the GDS successfully discerns diverse phases of dementia, especially from Phase 3 to Phase 6. ${ }^{[36]}$

\section{Discussion}

Clearly, the use of hospice for $\mathrm{NH}$ residents with dementia is low. ${ }^{[7]}$ Undoubtedly, hospice enrollment of $\mathrm{NH}$ residents with dementia has improved the quality at the end of life for dying residents compared to nonenrollees from previous studies. However, one major problem is that the guidelines to assess hospice for $\mathrm{AD}$ are less well identified than guidelines for other diseases. ${ }^{[20]}$ Although the $\mathrm{NHO}^{[22]}$ developed the guideline for cancer or noncancer-related diseases, medical guidelines to help professionals assess the 6-month prognosis of Alzheimer-type dementia were less well defined in clinical parameters. ${ }^{[20]}$

The most urgent problem is that Medicare currently requires the FAST tool for admission to hospice programs, despite the many flaws of the FAST, including that it does not originate from empirical data and is unable to linearly process dementia characteristics. It was also proposed that hospice nurses use the AHOPE rather than the FAST. ${ }^{[20]}$ However, Medicare guidelines require physicians' certification 
of 6-month survival of dementia patients. Extant literature indicates that expertly trained hospice nurses can assess 6-month survival with appropriate tools, but calls for further studies. The change in current Medicare guidelines may support the inclusion of hospice expert nurses in the 6-month evaluation for survival of dementia patients.

Another issue is the accessibility of tools. As mentioned earlier, the AHOPE, which proved more accurate than the FAST, was not accessible despite an exhaustive search. It is questionable how nurses in clinical settings can use a valid tool like the AHOPE. The use of inappropriate predicting tools and underuse of predicting tools is highly related to the very vulnerable population of hospice beneficiaries, which means that many dementia patients might not be provided adequate optimal palliative care before they die. Consequently, a more exact multidimensional analytical measure is required to determine the appropriate time for hospice care for AD patients. ${ }^{[37]}$ Few studies have offered statistical models to predict 6-month survival for persons with $\mathrm{AD} .^{[15]}$

Major problems for dementia patients include underuse of hospice care and nonexisting guidelines to assess hospice in patients with dementia and AD in Korea. The findings from a systematic review require that the scientific development of Korean assessment instruments predict the applicability of hospice care for elders with dementia. Future researchers should conduct studies to observe (1) longer ranges of time of survivors, (2) significant incident variables and assessment tools, (3) culturally sensitive appropriateness of hospice care for dementia patients, and (4) methodological modifications. ${ }^{[20]}$ Also, further studies should address methodological implications. Little research on innovation and interventions exists to predict the need for hospice care for elders with dementia in Korea. The application and evaluation of developed assessment tools to predict eligibility for hospice for Korean elders is urgent. Current hospice expert nurses may wish to translate and revise instruments, based on the instruments described above-AHOPE, ADEPT, FAST, GDS—and U.S. Medicare guidelines.

Successfully categorizing elders with dementia as hospice enrollees in Korea will improve the quality of end-of-life care for increasing numbers of elders with dementia and their family members. The use of inappropriate prediction tools and underuse of prediction tools is of great significance to the very vulnerable population of hospice beneficiaries. Many elders with dementia in Korea may not be provided optimal palliative care before they die. Consequently, more exact multidimensional analytical measures to determine the appropriate time for hospice care for $\mathrm{AD}$ elders in Korea are needed. ${ }^{[37]}$

\section{Acknowledgements}

This research was supported by Basic Sciene Research Program through the National Research Foundation of Korea (NRF) funded by the Ministry of Science, ICT \& Future Planning(grant \# 2-2014-1221-001-1).

\section{References}

[1] Allen RS, Kwak J, Lokken KL, et al. End-of-life issues in the context of Alzheimer's disease. Alzheimers Care Q. 2003; 4(4): 312 330. PMid:19997523.

[2] Alvarez-Fernandez B. Estimating prognosis for nursing home residents with advanced dementia. JAMA. 2004; 292: 1553-1554. http://dx.doi.org/10.1001/jama.292.13.1553-a

[3] Brown MA, Sampson EL, Jones L, et al. Prognostic indicators of 6-month mortality in elderly people with advanced dementia: A systematic review. Palliative Med. 2013; 27: 389-400. http: //dx.doi.org/10.1177/0269216312465649

[4] Choi SH, Na DY, Lee BH, et al. Validity of the Global Deterioration Scale. Korean J Clin Neurol. 2012; 20: 612-617.

[5] Christakis NA, Escarce JJ. Survival of Medicare patients after enrollment in hospice programs. New Engl J Med. 1996; 335: 172 178. http://dx.doi.org/10.1056/NEJM199607183350306

[6] Daly JM, Jones JK, Gereau PL, et al. Nonresponse error in mail surveys: top ten problems. Nursing Research and Practice. 2011; 2011: 987-924. http://dx.doi.org/10.1155/2011/987924

[7] Hanrahan P, Luchin DJ. Access to hospice programs in end-stage dementia: a national survey of hospice programs. J Am Geriatr Soc. 1995; 42: 56-59.

[8] Hartamier SL, Sloane PD, Guess HA, et al. The MDS cognition scale: A valid instrument for identifying and staging nursing home residents with dementia using the minimum data set. J Am Geriatr Soc. 1994; 42: 1173-1179.
[9] Johnson KS, Elbert-Avila K, Kuchibhatla M, et al. Characteristics and outcomes of hospice enrollees with dementia discharged alive. $\mathbf{J}$ Am Geriatr Soc. 2012; 60: 1638-1644. http://dx.doi .org/10. $1111 / \mathrm{j} .1532-5415.2012 .04117 . \mathrm{x}$

[10] Kiely DK, Givens JL, Shaffer ML, et al. Hospice use and outcomes in nursing home residents with advanced dementia. J Am Geriatr Soc. 2010; 58: 2284-91. http://dx.doi.org/10.1111/j.153 2-5415.2010.03185.x

[11] Kim H, Kwon S, Yoon NH, et al. Utilization of long-term care services under the public long-term care insurance program in Korea: Implications of a subsidy policy. Health Policy. 2013; 111: 166-174. http://dx.doi.org/10.1016/j.healthpol.2013.04.009

[12] Kim HC. Assessment of clinical progression in Alzheimer's disease with special reference to global deterioration scale (GDS) and functional assessment staging (FAST). J Korean Soc Biol Ther Psychiatry. 2002; 8: 354-366.

[13] Kinzbrunner B, Weinreb N, Policzer JS. 20 common problems: endof-life care. New York: McGraw-Hill; 2002.

[14] Korean Ministry of Health and Welfare. Recent increase of elderly with dementia in Korea. Seoul. 2013. Available from: http://www.nhis.or. kr/cms/board/board/Board.jsp?a $c t=V I E W \&$ communityKey=B0039\&boardId=22662 (13Septem ber2014, datelastaccessed)

[15] Korean National Health Insurance Corporation. Available from: http://www.nhic.or.kr/portal/site/main(21December 2013, datelastaccessed)

[16] Korean Nurses Association. Spiritual hospice. Seoul: Korean Nurses Association; 2002. 
[17] Korean Statistical Information Service. The death rates. Seoul [Internet]. 2013. Avaible from: http://kosis.kr/ups/ups_01List0 1.jsp?pubcode=YD (13September2014, datelastaccessed)

[18] Luchins DJ, Hanrahan P. What is appropriate health care for endstage dementia? J Am Geriatr Soc. 1993; 41: 25-30. PMid:7678104.

[19] Luchins DJ, Hanrahan P, Murphy K. Criteria for enrolling dementia patients in hospice. J Am Geriatr Soc. 1997; 45: 1054-1059. PMid:9288011.

[20] Marsh GW, Prochoda KP, Pritchett E, et al. Predicting hospice appropriateness for patients with dementia of the Alzheimer's type. Appl Nurs Res. 2000; 13: 187-196. http://dx.doi.org/10.10 53/apnr.2000.7654

[21] Medical guidelines for determining prognosis in selected non-cancer diseases. Hospice J. 1996; 11(2): 47-63. PMid:8949013.

[22] Miller SC, Lima JC, Mitchell SL. Influence of hospice on nursing home residents with advanced dementia who received Medicareskilled nursing facility care near the end of life. J Am Geriatr Soc. 2012; 60: 2035-2041. http://dx.doi.org/10.1111/j.1532-5 $415.2012 .04204 . \mathrm{x}$

[23] Miller SC, Lima JC, Mitchell SL. Hospice care for persons with dementia: the growth of access in US nursing homes. Am J Alzheimers Dis Other Demen. 2010; 25: 666-673. http://dx.doi.org/10. $1177 / 1533317510385809$

[24] Mitchell SL, Kiely DK, Hamel MB. Dying with advanced dementia in the nursing home. Arch Intern Med. 2004; 164: 321-356. http://dx.doi.org/10.1001/archinte.164.3.321

[25] Mitchell SL, Kiely DK, Hamel MB, et al. Estimating prognosis for nursing home residents with advanced dementia. JAMA. 2004; 291: 2734-2740. http://dx.doi.org/10.1001/jama.291.22 .2734

[26] Mitchell SL, Kiely DK, Miller SC, et al. Hospice care for patients with dementia. J Pain Symptom Manage. 2007; 34: 7-16. http: //dx.doi.org/10.1016/j.jpainsymman.2007.01.003

[27] Mitchell SL, Miller SC, Teno JM, et al. The advanced dementia prognostic tool: a risk score to estimate survival in nursing home residents with advanced dementia. J Pain Symptom Manage.
2010; 40: 639-651. http://dx.doi.org/10.1016/j.jpainsy mman.2010.02.014

[28] Mitchell SL, Miller SC, Teno JM, et al. Prediction of 6-month survival of nursing home residents with advanced dementia using ADEPT vs. hospice eligibility guidelines. JAMA. 2010; 304: 19291935. http://dx.doi.org/10.1001/jama.2010.1572

[29] $\mathrm{Na} \mathrm{HR}$, Kim SY, Chang YH, et al. Functional assessment staging (FAST) in Korean patients with Alzheimer's disease. J Alzheimers Dis. 2010; 22: 151-158. PMid:20847407.

[30] Reisberg B, Ferris SH, De-Leon MJ, et al. The global deterioration scale for assessment of primary degenerative dementia. Am J Psychiatry. 1982; 139: 1136-1139. PMid:7114305.

[31] Reisberg B, Jamil IA, Khan S, et al. Staging dementia. In Principles and Practice of Geriatric Psychiatry, MT Abousaleh C Katona, A Kumar, eds. Chichester, England: John Wiley and Sons. 2011; 162-169.

[32] Samala RV, Galindo DJ, Ciocon JO. Transitioning nursing home patients with dementia to hospice care: basics, benefits, and barriers. Ann Long Term Care. 2011; 19(4): 41-47.

[33] Schonwetter RS, Han B, Small BJ, et al. Predictors of six-month survival among patients with dementia: An evaluation of hospice Medicare guidelines. Am J Hosp Palliat Care. 2003; 20(2): 105-13. http://dx.doi.org/10.1177/104990910302000208

[34] Teno JM, Gozalo PL, Lee IC, et al. Does hospice improve quality of care for persons dying from dementia? J Am Geriatr Soc. 2011; 59: 1531-1536. http://dx.doi.org/10.1111/j.1532-5415. $2011.03505 . \mathrm{x}$

[35] Xie J, Brayne C, Matthews FE. Medical Research Council Cognitive, Function and Ageing Study Collaborators. Survival times in people with dementia: Analysis from population based cohort study with 14 year follow-up. BMJ. 2008; 336: 258-262. http: //dx.doi.org/10.1136/bmj .39433.616678.25

[36] Yoo HS. A study on the hospice service in elderly welfare facilities. Seoul: Sahmyook University; 2008.

[37] Yoon MO. The spiritual well-being and the spiritual nursing care of nurses for cancer patients. Korean J Hosp Palliat Care. 2009; 12: 72-79. 